\title{
Association between Working Hours and Anxiety/Depression of Medical Staff during Large-Scale Epidemic Outbreak of COVID-19: A Cross-Sectional Study
}

\author{
Qin Lang ${ }^{1}$, Xiaojing $\mathrm{Liu}^{2}$, Ying $\mathrm{He}^{3 凶}$, Qin $\mathrm{Lv}^{1}$, and Sibo $\mathrm{Xu}^{4}$ \\ ${ }^{1}$ Department of Pulmonary Diseases and Critical Care, Sichuan Academy of Medical Science \& Sichuan Provincial People's Hospital, Chengdu, China \\ 2Division of Pulmonary Diseases and Critical Care, The Affiliated Hospital of Qingdao University, Qingdao, China \\ ${ }^{3}$ Center of Psychosomatic Medicine, Sichuan Academy of Medical Science \& Sichuan Provincial, People's Hospital, Chengdu, China \\ ${ }^{4}$ Clinical Medical College of Qingdao University, Qindao, China
}

\begin{abstract}
Objective This present study aimed to investigate the relationship between working hours and anxiety/depression mood of medical staff in China during COVID-19 epidemic.

Methods The cross-sectional interview study was conducted during the period between February 14th and February 29th, 2020. A total of 291 Chinese medical professionals were recruited from 4 cities and participated in the study.

Results In 291 participants, $116(40.0 \%)$ medical staff experienced anxiety and 151 (51.8\%) underwent depressed mood. In male, the level of GAD-7 and PHQ-9 scores increased with the elevation of working hours per day (WHPD) $(\beta=0.579, p=0.003$ and $\beta=0.943$; $\mathrm{p}=0.001$ ) respectively. In female, nonlinear relationship mode was demonstrated. The levels of GAD-7 and PHQ-9 scores increased with the elevation of working hours when it was above 5 hours $(\beta=1.432 ; \mathrm{p}<0.001$ and $\beta=1.177 ; \mathrm{p}<0.001)$, but it did not have a significant association with WHPD when it was less than 5 ( $\mathrm{p}>0.05)$.

Conclusion During the COVID-19 epidemic, we found a strong correlation between the psychological mood and WHPD. The correlation followed different modes in male and female medical workers. Enforcing an upper time limit of WHPD may help decrease the risk of pandemic-related psychological problems in medical workers.

Psychiatry Investig 2020;17(12):1167-1174
\end{abstract}

Key Words Anxiety, Depression, COVID-19, Medical staff, Working hours.

\section{INTRODUCTION}

The Coronavirus Disease 2019 (COVID-19), firstly reported towards the end of 2019, has emerged as a new type of corona-virus infection with varied clinical symptoms ranging from a symptomatic state to lethal lung infection and multiorgan dysfunction. Similar to the previous outbreak in 2003severe acute respiratory syndrome (SARS), both COVID-19 and SARS were caused by a highly contagious corona-virus infection. The risk of infection is well known to be significant-

Received: June 16, 2020 Revised: September 4, 2020

Accepted: September 9, 2020

$\triangle$ Correspondence: Ying He, MD

Center of Psychosomatic Medicine, Sichuan Academy of Medical Science \& Sichuan Provincial People's Hospital, 32\# W. Sec 2, 1st Ring Rd. Chengdu, China Tel: +86-028-87393927, Fax: +86-028-87393927, E-mail: heyinger@126.com

(c) This is an Open Access article distributed under the terms of the Creative Commons Attribution Non-Commercial License (https://creativecommons.org/licenses/by$\mathrm{nc} / 4.0$ ) which permits unrestricted non-commercial use, distribution, and reproduction in any medium, provided the original work is properly cited. ly increased by interpersonal contact, especially amongst the front-line medical staff.

During the SARS epidemic outbreak, the medical staff accounted for up to $20 \%$ of the 5,327 confirmed cases in mainland China. ${ }^{1}$ Meanwhile, by February 11 , a total of 1,716 medical staff were diagnosed with COVID-19. ${ }^{2}$ This high prevalence was partly due to the lack of personal protection equipment as well as the lack of improper knowledge and preparedness with regards to preventive measures against the infection.

Although the Chinese government had taken active measures, such as sending more than 20,000 medical personnel from other cities to support the health system in Hubei province. It proved impossible to completely control the spread of infection in a short timeframe due to the highly contagious nature, the lack of vaccine and specific treatment. Medical staff were therefore exposed to an increased risk of intrahospital transmission as well as negative emotions such as fear, anxiety, and depression due to the increased work pressure. 
Studies showed that during the SARS epidemic in 2003, the risk of depression in the SARS ward nurses was significantly higher than others. ${ }^{3,4}$ Some of medical workers even experienced elevated stress levels and concerning high levels of psychological distress one year later. ${ }^{5}$ The psychological distress would negatively impact the work efficiency of health care providers. Therefore, the prevention and early intervention of psychological health problems in the medical staff are even more important at these challenging times.

According to a large sample of general population totaling $52,730,35 \%$ of responders had obvious stress emotional during the COVID-19 epidemic, ${ }^{6}$ indicating that the psychological health problems among the medical staff were more obvious than the ordinary group.?

In our systematic review, we learned that working long hours is associated with depressive state, anxiety mood, sleep disturbances, and even coronary heart disease. ${ }^{8}$ The anxiety and depressive symptoms were shown to be associated with long working hours in Chinese physicians. ${ }^{9}$ In this retrospective study, we investigated the anxiety and depression status of medical workers in four large cities in China and analyzed the association between working hours per day (WHPD) and anxiety/depression mood amongst medical staff.

\section{METHODS}

\section{Study design and participants}

This study was designed as a web-based cross-sectional survey which was broadcasted through the Wechat public platform by using a snowball sampling method. The participants in the study were medical professionals working in four cities: Wuhan, Chengdu, Shanghai, and Qingdao, who were invited to take part in the survey. The study participants were assessed following the eligibility criteria described as follows:

\section{Inclusion criteria}

1) Working in tertiary hospitals in China in the following job categories: (a) Doctors; (b) Nurses; (c) Other occupations, which included pharmacists, respiratory therapists, and support crew.

2) Consenting to survey during February 14th to February 29th, 2020.

\section{Exclusion criteria}

1) Incomplete answers in the survey questionnaires

2) Questionnaires that were completed $<1$ minute or $>60$ minutes would be excluded.

To ensure the high-quality data collection, the participants engagement with the survey was encouraged by providing them with information regarding the importance of investi- gation as well as explanatory commentaries for all questions throughout the questionnaire. As a result, a total of 313 medical professionals participated in the study, out of which 291 provided eligible responses with complete information based on the eligibility criteria described above, i.e., $93 \%$ response rate.

\section{Ethical statement}

The study protocol was approved by the Ethics Committee of Sichuan Provincial People's Hospital and the study was performed in complied with the Helsinki Declaration of ethical principles for medical research involving human subjects. Electronic informed consents were obtained from participants before starting the investigation. Participants also informed that they were free to withdraw from the survey at any moment without providing any justification (The approval number was Ethics Committee of Sichuan Provincial People’s Hospital 2020110).

\section{Data collection}

Data in this study was collected using the questionnaires as described below. Participants demographic variables were collected which included gender, age, and occupation categories. In addition, we also collected information related to work arrangements by including the following questions: 1) Do you have direct contact with confirmed COVID-19 patients? 2) Is there any family member or your friend diagnosed with COVID-19 infection? 3) How many days do you work every week? 4) How long do you work every day? 5) Do you work in Wuhan? 6) Do you have proper medical protection? 7) Do you live alone or not?

In order to collect information pertaining the anxiety and depression associated symptoms, specific questionnaires were used in line with the common practice, as described below.

Patient Health Questionnaire-9 (PHQ-9) for Depression symptoms had been previously used in Chinese population, with good reliability. The questionnaire comprises of nine areas of assessment which allowed the evaluation of depression symptoms severity over the past two weeks on a 4-point liker-scale ranging from 0 (never) to 3 (nearly every day). The total score of PHQ-9 ranged from 0 to 27, with increasing scores indicating severity for depression: $0-4$ none, 5-9 mild, $10-14$ moderate, $15-19$ moderately severe, $20-27$ severe. In our study, the presence of depression symptoms were defined by a total score of no less than 7 (7 points or greater). ${ }^{10}$

Generalized Anxiety Disorder (GAD-7) Anxiety mood symptoms were assessed using the 7-item GAD-7 questionnaire. The seven lines of assessment evaluated the frequency of anxiety symptoms over the past two weeks on a 4-point liker-scale ranging from 0 (never) to 3 (nearly every day). The total score of GAD-7 ranged from 0 to21. Severity for anxiety according 
to $\mathrm{GAD}-7: 0$ to $4=$ none.5-9=mild, $10-13=$ moderate, $14-18=$ moderate severe, $19-21=$ Severe. The GAD-7 has been previously used in Chinese population, and was shown to have good reliability. ${ }^{11}$ In our study, we defined a total score of no less than 9 as the presence of anxiety symptoms. ${ }^{12}$

\section{Statistical analysis}

Data were analyzed with the use of the statistical packages $\mathrm{R}$ (The R foundation; http://www.r-project.org;version3.4.3) and Empower(R) www.empowerstates.com. Data were presented as mean \pm standard deviation (SD), or median (inter quartile) for continuous variables, and as percentage for categorical variables. Statistical differences between the means and proportions of two groups was determined using the Mann-Whitney and chi-square tests. Binary logistic regression used to analyze the risk factors of anxiety and depressive mood (adjusted for age, gender, and other covariates with $\mathrm{p}<0.2$ in univariate analysis). Interaction and stratified analyses were conducted according to age, gender, working location, whether relatives or friends got COVID-19, living alone or not, working days ( $<4$ days or $\geq 4$ days), protection level and occupation. A multi-linear regression, a multi-piecewise linear regression, and a binary logistic regression were also applied to assess the independent correlation between WHPD and GAD-9/PHQ-7scores. P-value of $<0.05$ was considered statistically significant. The relationship between working hours per day and GAD-9/PHQ-7 scores was finally explored by a smooth curve fitting after adjustment for potential confounders.

\section{RESULTS}

\section{Demographic characteristics}

Data comprising the demographic characteristics, living status and job categories were collected from the study participants and presented in Table 1 . From the total of 313 questionnaires were distributed, 291 (92.9\%) were found eligible and subsequently analyzed. The median age of all participants was 33 (range 29-38) years old out of which 55 (18.9\%) were males and $236(81.1 \%)$ females. With regards to their occupation it was noted that $117(40.2 \%)$ were doctors, $102(35.1 \%)$ were nurses, and $72(24.7 \%)$ other occupations. Interestingly, we found that $66(22.7 \%)$ medical staff were enrolled from Wuhan, 122 (41.9\%) had contact with COVID-19 infected patients and 50 (17.2\%) had relatives or friends diagnosed COVID-19. Until the end of our study, none of those participants were reported infected to be diagnosed with COVID-19.

\section{Severity of measurements}

Analysis of the data obtained from the GAD-7 for anxiety and the PHQ-9 questionnaires for depression in the 291 re- spondents revealed a median (IQR) score of $6.0(3.0-11.0)$ and 7.0 (3.0-11.0), respectively total score of no less than 9 in GAD indicated psychological anxiety mood while a total score of no less than 7 in PHQ suggested psychological depression mood. As such, the data showed that $116(40.0 \%)$ of the medical staff was reported to experience anxiety mood and 151 (51.8\%) depression mood.

\section{Correlation between psychological scores (GAD-7 and PHQ-9) and demographic data, living or working related answers}

A univariate analysis was performed to analyze the relationship between each variable and the anxiety mood. As shown in Table 1, medical staff that had anxiety mood tended to be female, had longer WHPD, longer working days per week, or had a relative or friend who had been diagnosed with COVID-19. Furthermore, data showed that nurses were more likely to experience anxiety mood than doctors. On the other hand, those who experienced depression mood symptoms tended to be older, had longer WHPH, had a relative or friend who had been diagnosed with COVID-19, and living in isolation after work. Following multivariable logistic regression analysis we determined that only gender, working hours ( $\geq 5$ hours) and occupation were related to anxiety mood while age, working hours and living alone were related to depression mood (Table 2).

\section{Stratified analysis of association between working hours and anxiety/depression mood}

Stratified analyses were then performed by multiple factors, specifically age ( $<30$ or $\geq 30$ years), gender and working location. We also included factors such as whether relatives or friends were diagnosed with COVID-19, whether the participants were living alone or not and how many days per week they were working ( $<4$ days or $\geq 4$ days), use of personal protection equipment levels and occupation. Following this analysis we determined that the impact of working hours on anxiety mood was only affected by gender (interactions $<0.05$ ) while the impact of working hours on the depression mood were only affected by working location (interactions $<0.05$ ) (Supplementary Table 1 and 2 in the online-only Data Supplement).

We were unable to use working hours as categorical variables in multivariable regression due to the small numbers of participants in our result. However, it is important to note that it may be inappropriate to use multi-variable regression if the continuous variable (working hours) and dependent variable were not in a lineal model. These results are therefore presented in Figures 1 and 2. 
Table 1. Demographic/occupational characteristics and univariate analysis

\begin{tabular}{|c|c|c|c|c|c|c|}
\hline & \multicolumn{3}{|c|}{ Anxiety } & \multicolumn{3}{|c|}{ Depression } \\
\hline & No & Yes & $\mathrm{p}$-value & No & Yes & $\mathrm{p}$-value \\
\hline Number & 175 & 116 & & 140 & 151 & \\
\hline Age & $34.0(29.0-40.0)$ & $32.0(28.8-38.0)$ & 0.307 & $32.0(27.0-37.0)$ & $34.0(30.0-40.0)$ & $0.001^{*}$ \\
\hline Gender (\%) & & & $0.006^{*}$ & & & 0.174 \\
\hline Male & $42(24.0)$ & $13(11.2)$ & & $31(22.1)$ & $24(15.9)$ & \\
\hline Female & $133(76.0)$ & $103(88.8)$ & & $109(77.9)$ & $127(84.1)$ & \\
\hline Working hours per day & $6.0(4.0-8.0)$ & $9.5(8.0-10.0)$ & $<0.001^{*}$ & $6.2(4.0-8.0)$ & $8.0(8.0-10.0)$ & $<0.001^{*}$ \\
\hline Working days per week & $5.0(4.0-5.0)$ & $5.0(4.0-6.0)$ & $0.063^{*}$ & $5.0(3.8-6.0)$ & $5.0(4.0-6.0)$ & 0.789 \\
\hline Location & & & 0.344 & & & 0.293 \\
\hline Wuhan (\%) & $43(24.6)$ & $23(19.8)$ & & $28(20.0)$ & $38(25.2)$ & \\
\hline Othercities (\%) & $132(75.4)$ & $93(80.2)$ & & $112(80.0)$ & $113(74.8)$ & \\
\hline Live alone (\%) & & & 0.851 & & & $0.049^{*}$ \\
\hline No & $121(69.1)$ & $79(68.1)$ & & $36(25.7)$ & $55(36.4)$ & \\
\hline Yes & $54(30.9)$ & $37(31.9)$ & & $104(74.3)$ & $96(63.6)$ & \\
\hline Relative or friend got COVID-19 (\%) & & & $<0.001^{*}$ & & & $0.028^{*}$ \\
\hline No & $156(89.1)$ & $85(73.3)$ & & $123(87.9)$ & $118(78.1)$ & \\
\hline Yes & $19(10.9)$ & $31(26.7)$ & & $17(12.1)$ & $33(21.9)$ & \\
\hline Patient exposure (\%) & & & 0.566 & & & 0.380 \\
\hline No & $104(59.4)$ & $65(56.0)$ & & $85(60.7)$ & $84(55.6)$ & \\
\hline Yes & $71(40.6)$ & $51(44.0)$ & & $55(39.3)$ & $67(44.4)$ & \\
\hline Protection (\%) & & & 0.271 & & & 0.235 \\
\hline Well & $32(18.3)$ & $19(16.4)$ & & $23(16.4)$ & $28(18.5)$ & \\
\hline Ordinary & $80(45.7)$ & $64(55.2)$ & & $64(45.7)$ & $80(53.0)$ & \\
\hline Poor & $63(36.0)$ & $33(28.4)$ & & $53(37.9)$ & $43(28.5)$ & \\
\hline Job category (\%) & & & $0.002^{*}$ & & & 0.321 \\
\hline Others & $54(30.9)$ & $18(15.5)$ & Reference & $40(28.6)$ & $32(21.2)$ & Reference \\
\hline Nurse & $49(28.0)$ & $53(45.7)$ & $<0.001^{*}$ & $48(34.3)$ & $54(35.8)$ & 0.792 \\
\hline Doctor & $72(41.1)$ & $45(38.8)$ & 0.057 & $52(37.1)$ & $65(43.0)$ & 0.305 \\
\hline
\end{tabular}

${ }^{*} \mathrm{p}<0.2$ (covariates with $\mathrm{p}<0.2$ in univariate analysis were put into binary logistic regression in Table 2 )

\section{The Independent Correlation between psychological scores and WHPD}

In the binary logistic regression the covariates screening criteria for possible confounders was found to be $\mathrm{p}<0.2$ in the univariate analysis. The results demonstrated that factors such as the number of working days per week, whether a relative or friend had been diagnosed with COVID-19 as well as job categories met the filter criteria when the outcome variable was represented by the GAD-7 scores. Similarly, whether the participant was living alone and whether a relative or friend had been diagnosed with COVID-19 met the filter criteria when the outcome variable was the PHQ-9 scores.

Finally, a smooth curve fitting was performed after adjustment for covariates and the resultant curve exhibited a twostage change and a breakpoint in females as shown in Figure
1 and 2. Namely, in females, there was a positive relationship between WHPD and GAD-7 scores when WHPD was above 5 hours $(\beta=1.432,95 \% \mathrm{CI}=1.151-1.713, \mathrm{p}<0.001)$ (Table 3$)$, while there was no significant association between those two variables when WHPD was less than 5 hours ( $\mathrm{p}>0.05)$. So did the association between WHPD and PHQ-9 scores, where the breaking point was represented by the WHPD equaling 5 hours was ( $\beta=1.177,95 \% \mathrm{CI}=0.877-1.476, \mathrm{p}<0.001$ ) (Table 4). In binary logistic regression, women who worked more than 5 hours per day were more likely to experience anxiety or depression $\operatorname{mood}(\mathrm{OR}=23.729,95 \% \mathrm{CI}=5.361-105.030, \mathrm{p}<0.001$; $\mathrm{OR}=4.981,95 \% \mathrm{CI}=2.200-11.276, \mathrm{p}<0.001$ ). For medical workers who were males there was no breakpoint found in the smooth curve fitting. In linear regression, both GAD-7 scores and PHQ-9 scores were positively associated with WHPD 
$(\beta=0.579,95 \% \mathrm{CI}=0.214-0.945, \mathrm{p}<0.05 ; \beta=0.886,95 \% \mathrm{CI}=$ $0.336-1.435$ ), $\mathrm{p}<0.05$ respectively) (Figures 1 and 2 ) and (Table 3 and 4$)$.

\section{DISCUSSION}

Until 2nd March, 2020, a total of 80,175 cases were con-

Table 2. Risk factors for mental health outcomes identified by binary logistic regression

\begin{tabular}{|c|c|c|c|}
\hline \multirow{2}{*}{ Variable } & \multirow{2}{*}{ Adjusted OR $(95 \% \mathrm{CI})^{\mathrm{a}}(95 \%$ confidence interval $)$} & \multicolumn{2}{|c|}{ p-value } \\
\hline & & Category $^{c}$ & Overall \\
\hline \multicolumn{4}{|l|}{ GAD-7, anxiety symptoms } \\
\hline Age & & & 0.660 \\
\hline$<30 y$ & Ref & NA & \\
\hline$\geq 30 y$ & $0.870(0.469,1.616)$ & 0.660 & \\
\hline \multicolumn{4}{|l|}{ Gender } \\
\hline Male & Ref & NA & 0.05 \\
\hline Female & $2.086(1.001,4.350)$ & 0.05 & \\
\hline \multicolumn{4}{|c|}{ Relative or friend got COVID-19 } \\
\hline No & Ref & NA & 0.066 \\
\hline Yes & $2.002(0.915,3.643)$ & 0.066 & \\
\hline Working hours per day & & & $<0.001$ \\
\hline$<5 \mathrm{~h}$ & Ref & NA & \\
\hline$\geq 5 \mathrm{~h}$ & $15.723(4.607,53.665)$ & $<0.001$ & \\
\hline Working days per week & & & 0.432 \\
\hline$<4$ days & Ref & NA & \\
\hline$\geq 4$ days & $0.771(0.403,1.474)$ & 0.432 & \\
\hline \multicolumn{4}{|l|}{ Job category } \\
\hline Others & Ref & NA & 0.04 \\
\hline Nurse & $2.691(1.246,5.809)$ & 0.012 & \\
\hline Doctor & $1.826(0.915,3.643)$ & 0.087 & \\
\hline
\end{tabular}

PHQ-9, depression symptoms

Age

$<30 \mathrm{y}$

Ref

$<0.001$

$\geq 30 \mathrm{y}$

$3.456(1.928,6.195)$

NA

Gender

Ref

Male

$1.675(0.881,3.184)$

0.116

Relative or friend got COVID-19

1.675 (0.881, 3.184$)$

Ref

No

$2.011(0.960,4.212)$

Yes

4.371 (2.051, 9.315)

$<0.001$

0.116

Working hours per day

$<5 \mathrm{~h}$

$\geq 5 \mathrm{~h}$

Live alone

No

Ref

$1.867(1.050,3.320)$

Yes

0.064

NA

0.064

NA

$<0.001$

NA

0.034

adjusted for age, gender, whether a relative or friend got COVID-19 or not, working days per week, WHPD, and job categories, ${ }^{\mathrm{b}}$ adjusted for age, gender, whether a relative or friend got COVID-19 or not, WHPD, and whether live alone, ${ }^{c}$ category refers to the $\mathrm{p}$ value for each category vs. the reference, while overall refers to the results of the logistic regression 
firmed, out of which 2915 died and 7,110 were seriously ill. The COVID-19 pandemic posed great pressure on healthcare systems leading to mental health problems amongst the frontline health care providers. These mental health issues affected not only the medical workers' attention, decision making ability, which might hinder the fight against COVID-19, but they could also have a lasting effect on their overall well-

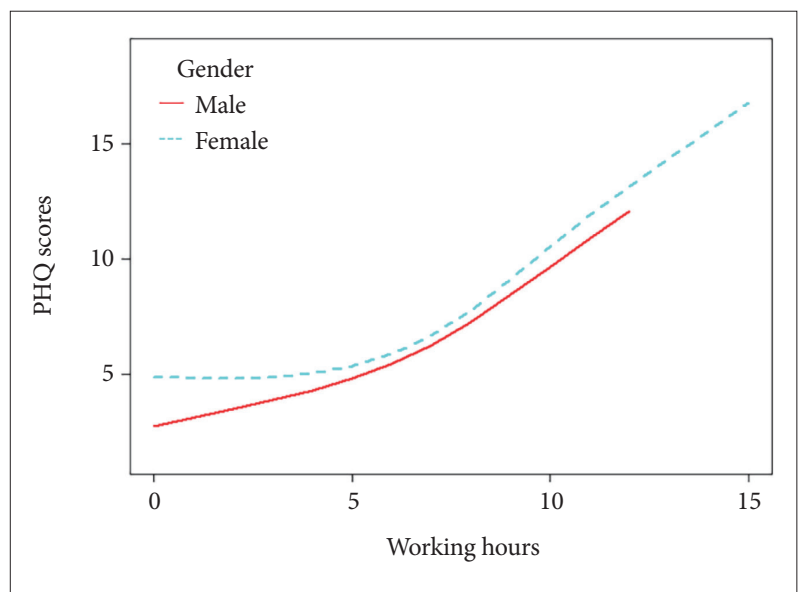

Figure 1. The relationship between GAD-7 SCORES and working time (hours/day) by smooth curve fitting in different genders. Adjusted variables: age, whether a relative or friend got COVID-19 or not, working days per week and job categories. being. ${ }^{13}$ Prior studies showed that health care providers may develop psychiatric disorders after coping with stressful community events. During the SARS outbreak in Singapore, 39.1\% of health care workers reported psychiatric symptoms. ${ }^{14}$ Similarly, during the Ebola outbreaks in Sierra Leone in 2014 and in the Democratic Republic of the Congo in 2018, medical staff reported high levels of anxiety and the impact of stigma. ${ }^{15}$ In

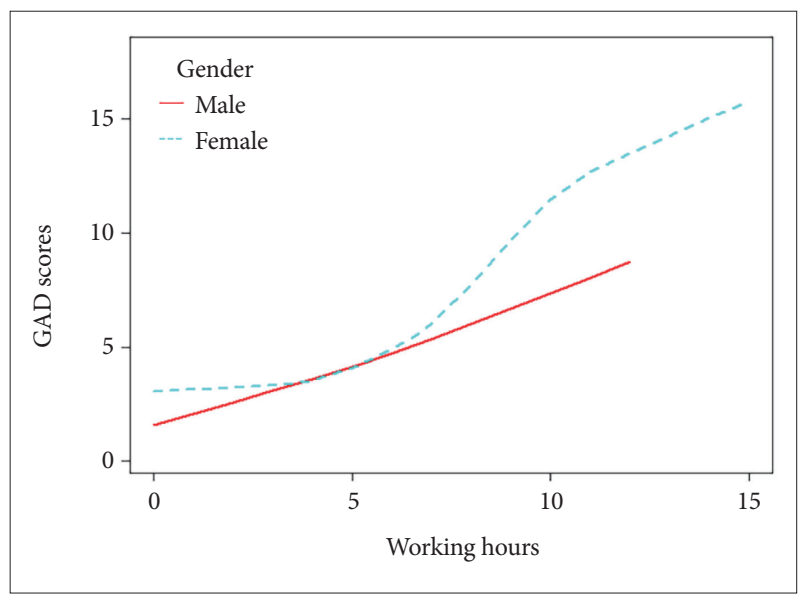

Figure 2. The relationship between PHQ SCORES and working time (hours/day) by smooth curve fitting in different genders. Adjusted variables: age, whether living in isolation or not, whether a relative or friend got COVID-19.

Table 3. The independent correlation between GAD-7 SCORES and WHPD in different gender

\begin{tabular}{|c|c|c|c|c|}
\hline \multirow{2}{*}{ Gender } & \multicolumn{2}{|l|}{ Male } & \multicolumn{2}{|l|}{ Female } \\
\hline & $\beta$ or OR $(95 \% \mathrm{CI})$ & $\mathrm{p}$ value & $\beta$ or OR $(95 \% \mathrm{CI})$ & $\mathrm{p}$ value \\
\hline Linear regression & $0.579(0.214,0.945)$ & $0.003^{*}$ & $1.159(0.942,1.375)$ & $<0.001^{*}$ \\
\hline \multicolumn{5}{|c|}{ Multivariable piecewise linear regression } \\
\hline$<5$ hours (section 1 ) & $-0.035(-0.808,0.738)$ & 0.929 & $0.062(-0.696,0.821)$ & 0.872 \\
\hline$>5$ hours $(\text { section } 2)^{\mathrm{a}}$ & $0.851(0.379,1.324)$ & $0.001^{*}$ & $1.432(1.151,1.713)$ & $<0.001^{*}$ \\
\hline Predicted value at 5 hours & $3.439(1.999,4.879)$ & & $3.516(2.425,4.608)$ & \\
\hline Binary logistic regression ${ }^{\mathrm{b}}$ & $4.125(0.246,69.298)$ & 0.324 & $23.729(5.361,105.030)$ & $<0.001^{*}$ \\
\hline
\end{tabular}

Adjusted variables: age, whether a relative or friend got COVID-19 or not, working days per week and job categories. ${ }^{*} \mathrm{p}<0.05$. ${ }^{a}$ models (sec-

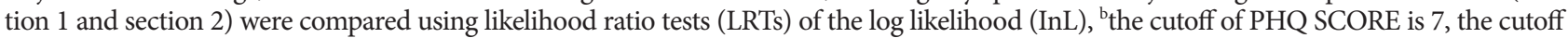
of working hour is 5 hours

Table 4. The independent correlation between PHQ-9 SCORES and WHPD in different gender

\begin{tabular}{|c|c|c|c|c|}
\hline \multirow{2}{*}{ Gender } & \multicolumn{2}{|l|}{ Male } & \multicolumn{2}{|l|}{ Female } \\
\hline & $\beta$ or OR $(95 \% \mathrm{CI})$ & $\mathrm{p}$ value & $\beta$ or OR $(95 \% \mathrm{CI})$ & $\mathrm{p}$ value \\
\hline Linear regression $^{\mathrm{a}}$ & $0.886(0.336,1.435)$ & $0.003^{*}$ & $0.860(0.634,1.087)$ & $<0.001^{*}$ \\
\hline \multicolumn{5}{|c|}{ Multivariable piecewise linear regression } \\
\hline$<5$ hours (section 1 ) & $0.208(-0.994,1.410)$ & 0.736 & $-0.321(-1.106,0.465)$ & 0.425 \\
\hline$>5$ hours (section 2 ) & $1.163(0.417,1.909)$ & $0.004^{*}$ & $1.177(0.877,1.476)$ & $<0.001^{*}$ \\
\hline Predicted value at 5 hours & $3.835(1.358,6.312)$ & & $4.148(2.941,5.354)$ & \\
\hline Binary logistic regression ${ }^{\mathrm{b}}$ & $2.434(0.370,16.011)$ & 0.355 & $4.981(2.200,11.276)$ & $<0.001^{*}$ \\
\hline
\end{tabular}

Adjusted variables: age, whether a relative or friend got COVID-19 or not and whether living in isolation. ${ }^{*} \mathrm{p}<0.05$. ${ }^{\mathrm{a}}$ models were compared

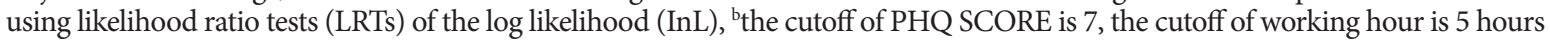


our research the overall prevalence of anxiety and depression symptoms were as high as $40.0 \%, 51.8 \%$ respectively, similar to another cross-sectional survey in which anxiety was reported in $44.6 \%$ and depression in $50.4 \%$ of the medical staff. ${ }^{16}$

In multivariable regression analysis, females, nurses, and WHPD $\geq 5$ h were more likely to develop anxiety symptoms. This study confirmed previous findings that female medical workers were more likely to have anxiety symptoms than males. This conclusion was not only found in medical staff, ${ }^{16,17}$ but also in general population. ${ }^{6,18}$ It also provided confirmation that nurses were more prone to suffer anxiety mood in comparison to other occupations. ${ }^{17,19}$ This may be explained by the fact that in China, the majority of nurses were women. Another possible reason could be that the shortage of nurses in pulmonary and critical care unit resulted in the frequent night shift rostering, which was associated with significantly higher scores on anxiety symptoms. ${ }^{20}$

Our results in this study revealed that subjects older than 30 years of age, living alone, and WHPD $\geq 5 \mathrm{~h}$ were more likely to develop depression mood. Older medical workers were prone to get depression mood. In contrast, another study showed that the prevalence of depressive symptoms was significantly higher in younger participants, especially in those who were under $35 .{ }^{21}$ The reason for this difference may be the narrower range of age in our study. Living alone may worsen the sense of loneliness and this may represent another risk factor to develop psychological depressive symptoms. Other researchers also found that living in isolation represented risk factors for nurturing negative emotions in health care providers. ${ }^{17,22}$ This may suggest that the face to face communication with the family members may provide emotional support and ease the depression mood. We found that neither the direct contact with COVID-19 patients, nor having relatives or friends infected were risk factors for depression mood. However, these findings were inconsistent with similar studies. ${ }^{20,21}$ This may be because our research was not carried out at the beginning of the outbreak. At the time of our research the shortage of personal protective equipment had largely been solved and experience of dealing with COVID-19 patients was significantly improved. The more we knew about the mode of transmission of COVID-19 infection and its effective treatments, the less panic this may cause. Furthermore, it should be noted that our relative small sampling may account for a false negative result. Whether those two factors may have an effect on the psychoemotional status of medical workers remains to be elucidated.

The most significant implication of this study concerns the relationship between WHPD and the negative emotions experienced by the healthcare providers. Consistent high intensity of work, conflicting time demands and heavy profession- al responsibility put the healthcare providers at high risk of detrimental psychological issues, but few researches had addressed the issue whether there should be a limited working time set in order to prevent burn out of the medical workers. Our study was consistent with the fact that working longer hours was associated with depressive and anxiety state shown in previous studies. ${ }^{8}{ }^{81}$ In Virtanen M's study, working no less than 11 hours a day was associated with a 2.3- to 2.5-fold risk of depression compared with a standard 7-8 hours. ${ }^{23}$ In Le Dang Khoa's research, ${ }^{24}$ long working hours, that were defined as more than 48 hours per week, also represented a significantly high source of job related stress. In our research, the level of GAD-7 and PHQ-9 scores increased proportionally with the elevation of WHPD, which fit the linear regression in male medical workers. Another finding was that working 5 hours per day were the turning point of being susceptible to anxiety/ depression mood for female medical workers. A plausible reason may be due to the different physical characteristics between genders. For example, female medical workers, during their menstrual cycle, felt more uncomfortable, especially when wearing PPE (such as positive-pressure exhaust suits). Furthermore, when faced with heavy manual work, it was possible that female medical workers feel fatigue in a different way. However, the underlying mechanism requires further research in order to be fully explored. It also highlighted the fact that we should consider the special needs for female medical health workers in this strict nosocomial infections prevention setting.

We observed in our study a possible interaction between depression mood and working location (Supplementary Table 1 and 2 in the online-only Data Supplement) Compared with peers in Wuhan, medical workers from other cities with longer WHPD were more susceptible to depression mood. This outcome was in contradiction with previous research. ${ }^{19}$ The possible mechanism was that an increased volume of medical resources were rushed into Wuhan to support the local health system during our survey period. Abundant medical resources may relief the stress and reduce the risk of psychological problems of medical staff. Future studies should examine the effects on the mental health of health care providers that medical resources have, such as the accessibility to appropriate testing methods for COVID-19 (polymerase chain reaction based tests), the availability of PPE and the ratio of medical workers to patients.

\section{Limitation}

We noted several limitations in our study. Firstly, our study used a rather small sample size which may not be representative of the general medical staff. Secondly, the snowball sampling technique, which is a non-random sampling method, 
may have led to sampling of participants sharing similar views and we were therefore unable to assess an individual's basic psychiatric and physical conditions before the outbreak and lack of follow-up investigations. Thirdly, the R2, a statistical measure of how close the data are to the fitted regression line, was only 0.245 for anxiety symptom and 0.202 for depression symptom $(24.5 \% / 20.2 \%$ of the variability of the response data around its mean). Thus, other potential factors associated with anxiety or depression mood remained to be elucidated.

\section{Supplementary Materials}

The online-only Data Supplement is available with this article at https://doi.org/10.30773/pi.2020.0229.

\section{Acknowledgments}

The author thanks Xiaowei Xing for help in the data analysis. Also special thanks to colleagues in the division of Pulmonary Diseases and Critical Care, Haiying Yan, Jia Yang, Kaiju Xu, Lei Deng, Duozi Wang, for the discussion of study protocol and distribution of questionnaires. Also, I would like to thank the medical staff who participate this research during their busy work.

\section{Conflicts of Interest}

The authors have no potential conflicts of interest to disclose.

\section{Author Contributions}

Conceptualization: Qin Lang, Ying He. Data curation: Qin Lang, Xiaojing Liu. Formal analysis: Ying He. Investigation: Qin Lang, Xiaojing Liu, Qin Lu. Methodology: Qin Lang. Project administration: Ying He. Resources: Qin Lu. Software: Qin Lang. Supervision: Ying He. Validation: Qin Lang. Visualization: Qin Lang. Writing_original draft: Qin Lang. Writing_review \& editing: Ying He.

\section{ORCID iDs}

$\begin{array}{ll}\text { Qin Lang } & \text { https://orcid.org/0000-0002-0381-2931 } \\ \text { Xiaojing Liu } & \text { https://orcid.org/0000-0003-4778-8299 } \\ \text { Ying He } & \text { https://orcid.org/0000-0002-3754-2482 } \\ \text { Qin Lv } & \text { https://orcid.org/0000-0002-8045-594X } \\ \text { Sibo Xu } & \text { https://orcid.org/0000-0003-2189-2733 }\end{array}$

\section{REFERENCES}

1. Huang G, Feng Y. Diagnosis and treatment of SARS. Chin J Respir Crit Care Med 2003;002:153-160.

2. Joint prevention and control mechanism for novel coronavirus pneumoni. Available at: http://www.nhc.gov.cn/xcs/s3574/202002/5329d7a b7af24690a1d5b66982333af3.shtml. Accessed February 14, 2020.

3. Nickell LA, Crighton EJ, Tracy CS, Al-Enazy H, Bolaji Y, Hanjrah S, et al. Psychosocial effects of SARS on hospital staff: survey of a large tertiary care institution. CMAJ 2004;170:793-798.

4. Chen CS, Wu HY, Yang P, Yen CF. Psychological distress of nurses in Taiwan who worked during the outbreak of SARS. Psychiatr Serv 2005;56: 76-79.

5. Lung FW, Lu YC, Chang YY, Shu BC. Mental symptoms in different health professionals during the SARS attack: a follow-up study. Psychiatr Q 2009;80:107-116.

6. Qiu J, Shen B, Zhao M, Wang Z, Xie B, Xu Y. A nationwide survey of psychological distress among Chinese people in the COVID-19 epi- demic: implications and policy recommendations. Gen Psychiatr 2020; 33:e100213.

7. Wang C, Pan R, Wan X, Tan Y, Xu L, Ho CS, et al. Immediate psychological responses and associated factors during the initial stage of the 2019 Coronavirus Disease (COVID-19) epidemic among the general population in China. Int J Environ Res Public Health 2020;17.

8. Bannai A, Tamakoshi A. The association between long working hours and health: a systematic review of epidemiological evidence. Scand J Work Environ Health 2014;40:5-18.

9. Gong Y, Han T, Chen W, Dib HH, Yang G, Zhuang R, et al. Prevalence of anxiety and depressive symptoms and related risk factors among physicians in China: a cross-sectional study. Plos One 2014;9:e103242.

10. Wang W, Bian Q, Zhao Y, Li X, Wang W, Du J, et al. Reliability and validity of the Chinese version of the Patient Health Questionnaire (PHQ-9) in the general population. Gen Hosp Psychiatry 2014;36:539-544.

11. He X, Li C, Qian J, Cui HS, Wu W. Reliability and validity of a generalized anxiety scale in general hospital outpatients. Shanghai Arch Psychiatry 2010;22:200-203.

12. Wang $Y$, Cheng $R$, Zhang 1 . Reliability and validity of generalized anxiety scale-7 in patients in Chinese general hospital. J Clin Psychiatr 2018;28: 168-171.

13. Kang L, Li Y, Hu S, Chen M, Yang C, Yang BX, et al. The mental health of medical workers in Wuhan, China dealing with the 2019 novel coronavirus. Lancet Psychiatry 2020;7:e14.

14. Chan AO, Huak CY. Psychological impact of the 2003 severe acute respiratory syndrome outbreak on health care workers in a medium size regional general hospital in Singapore. Occup Med (Lond) 2004;54:190196.

15. Lee SM, Kang WS, Cho AR, Kim T, Park JK. Psychological impact of the 2015 MERS outbreak on hospital workers and quarantined hemodialysis patients. Compr Psychiatry 2018;87:123-127.

16. Zhang WR, Wang K, Yin L, Zhao WF, Xue Q, Peng M, et al. Mental health and psychosocial problems of medical health workers during the COVID-19 epidemic in China. Psychother Psychosom 2020;89:242-250.

17. Luo M, Guo L, Yu M, Jiang W, Wang H. The psychological and mental impact of coronavirus disease 2019 (COVID-19) on medical staff and general public - A systematic review and meta-analysis. Psychiatry Res 2020;291:113190.

18. Virtanen M, Ferrie JE, Singh-Manoux A, Shipley MJ, Stansfeld SA, Marmot MG, et al. Long working hours and symptoms of anxiety and depression: a 5-year follow-up of the Whitehall II study. Psychol Med 2011; 41:2485-2494

19. Lai J, Ma S, Wang Y, Cai Z, Hu J, Wei N, et al. Factors associated with mental health outcomes among health care workers exposed to coronavirus disease 2019. JAMA Netw Open 2020;3:e203976.

20. Kang MY, Kwon HJ, Choi KH, Kang CW, Kim H. The relationship between shift work and mental health among electronics workers in South Korea: a cross-sectional study. PLoS One 2017;12:e0188019.

21. Su TP, Lien TC, Yang CY, Su YL, Wang JH, Tsai SL, et al. Prevalence of psychiatric morbidity and psychological adaptation of the nurses in a structured SARS caring unit during outbreak: a prospective and periodic assessment study in Taiwan. J Psychiatr Res 2007;41:119-130.

22. Dai Y, Hu G, Xiong H, Qiu H, Yuan X. Psychological impact of the coronavirus disease 2019 (COVID-19) outbreak on healthcare workers in China. MedRxiv 2020 Prepirnt.

23. Virtanen M, Stansfeld SA, Fuhrer R, Ferrie JE, Kivimaki M. Overtime work as a predictor of major depressive episode: a 5-year follow-up of the Whitehall II study. PLoS One 2012;7:e30719.

24. Khoa LD, Quang TN, Vinh DQ, Anh NTN, Tuong HM Foster K. The prevalence of job stressors among nurses in private in vitro fertilization (IVF) centres. Nurs Open 2019;6:39-49. 
Supplementary Table 1. Associations between anxiety status and WHPD in different subgroups

\begin{tabular}{|c|c|c|c|}
\hline WHPD & Crude & Adjusted & $\mathrm{p}$ for interaction \\
\hline Gender & & & 0.0694 \\
\hline Male & $1.44(1.07,1.93)$ & $1.44(1.04,1.99)$ & \\
\hline Female & $2.00(1.65,2.41)$ & $2.08(1.69,2.55)$ & \\
\hline Age & & & 0.3694 \\
\hline$<30 \mathrm{y}$ & $1.77(1.38,2.28)$ & $1.74(1.32,2.30)$ & \\
\hline$\geq 30 y$ & $1.98(1.61,2.44)$ & $2.05(1.63,2.56)$ & \\
\hline Relative or friends got COVID-19 & & & 0.5611 \\
\hline No & $1.89(1.57,2.27)$ & $1.70(1.25,2.30)$ & \\
\hline Yes & $1.99(1.62,2.45)$ & $1.71(1.26,2.32)$ & \\
\hline Location & & & 0.2411 \\
\hline Other cities & $1.96(1.62,2.37)$ & $1.59(1.20,2.11)$ & \\
\hline Wuhan & $2.03(1.65,2.50)$ & $1.62(1.20,2.20)$ & \\
\hline Occupation & & & 0.1042 \\
\hline Doctors & $1.75(1.36,2.25)$ & $1.76(1.34,2.31)$ & \\
\hline Nurses & $1.75(1.40,2.19)$ & $1.79(1.40,2.28)$ & \\
\hline Others & $2.84(1.64,4.95)$ & $3.17(1.75,5.75)$ & \\
\hline Protection & & & 0.2513 \\
\hline Well & $1.99(1.30,3.04)$ & $1.80(1.18,2.75)$ & \\
\hline Normal & $1.95(1.52,2.49)$ & $2.12(1.62,2.77)$ & \\
\hline Poor & $1.71(1.35,2.17)$ & $1.76(1.36,2.27)$ & \\
\hline Patients exposure & & & 0.2982 \\
\hline No & $1.85(1.50,2.29)$ & $1.86(1.47,2.36)$ & \\
\hline Yes & $1.76(1.41,2.21)$ & $2.09(1.61,2.71)$ & \\
\hline Isolation & & & 0.3058 \\
\hline No & $2.10(1.56,2.82)$ & $1.76(1.45,2.13)$ & \\
\hline Yes & $2.02(1.52,2.69)$ & $1.86(1.50,2.31)$ & \\
\hline
\end{tabular}


Supplementary Table 2. Associations between depression status and WHPD in different subgroups

\begin{tabular}{|c|c|c|c|}
\hline WHPD & Crude & Adjusted & \multirow{2}{*}{$\mathrm{p}$ for interaction } \\
\hline Subgroups, hours/day (no) & OR $(95 \% \mathrm{CI})$ & OR $(95 \%$ CI $)$ & \\
\hline \multicolumn{4}{|l|}{ Gender } \\
\hline Male & $1.36(1.07,1.73)$ & $1.36(1.05,1.77)$ & 0.9616 \\
\hline Female & $1.37(1.22,1.54)$ & $1.39(1.23,1.57)$ & \\
\hline Age & & & 0.8173 \\
\hline$<30 y$ & $1.34(1.12,1.61)$ & $1.36(1.12,1.65)$ & \\
\hline$\geq 30 y$ & $1.37(1.21,1.56)$ & $1.40(1.22,1.60)$ & \\
\hline \multicolumn{4}{|l|}{ Relative or friends got COVID-19 } \\
\hline No & $1.34(1.20,1.50)$ & $1.34(1.19,1.51)$ & 0.7259 \\
\hline Yes & $1.47(1.13,1.91)$ & $1.38(1.04,1.83)$ & \\
\hline Location & & & 0.0102 \\
\hline Other cities & $1.47(1.29,1.68)$ & $1.48(1.29,1.70)$ & \\
\hline Wuhan & $1.18(0.98,1.41)$ & $1.08(0.89,1.32)$ & \\
\hline Occupation & & & 0.2262 \\
\hline Doctors & $1.21(1.03,1.42)$ & $1.21(1.03,1.43)$ & \\
\hline Nurses & $1.55(1.28,1.88)$ & $1.51(1.23,1.86)$ & \\
\hline Others & $1.38(1.12,1.70)$ & $1.40(1.12,1.74)$ & \\
\hline Protection & & & 0.9786 \\
\hline Well & $1.44(1.11,1.87)$ & $1.32(1.01,1.71)$ & \\
\hline Normal & $1.34(1.16,1.56)$ & $1.33(1.14,1.56)$ & \\
\hline Poor & $1.37(1.15,1.62)$ & $1.38(1.15,1.65)$ & \\
\hline Patients exposure & & & 0.7877 \\
\hline No & $1.39(1.21,1.59)$ & $1.36(1.18,1.57)$ & \\
\hline Yes & $1.36(1.18,1.57)$ & $1.33(1.11,1.58)$ & \\
\hline Isolation & & & 0.3756 \\
\hline No & $1.35(1.14,1.58)$ & $1.26(1.06,1.51)$ & \\
\hline Yes & $1.39(1.21,1.60)$ & $1.40(1.22,1.61)$ & \\
\hline
\end{tabular}

